\title{
Immunity following use of Australian tick fever vaccine: a review of the evidence
}

\author{
RE BOCK and AJ DE VOS \\ Tick Fever Research Centre, Animal and Plant Health Service, Q ueensland Department of Primary Industries, 280 Grindle Road, \\ Wacol, Queensland 4076, E-mail russell.bock@dpi.qld.gov.au
}

\begin{abstract}
Objective To review the evidence available on the degree and duration of immunity provided by Australian tick fever vaccines against Babesia bovis, $B$ bigemina and Anaplasma marginale infections in Australia and overseas.
\end{abstract}

Background Vaccines containing attenuated strains of $B$ bovis and $B$ bigemina as well as $A$ centrale grown in splenectomised calves have been used in Australia since 1964 to immunise cattle against tick fever. About 800,000 doses of vaccine are supplied annually and much of the evidence for protection is field evidence rather than conventional immunological measures or pen trials.

\section{Conclusions}

Immunity to Babesia bovis and B bigemina - A single inoculation generally provides sound, long-lasting protection both in Australia and overseas. No evidence was found of a loss of immunity with time. Vaccine failures to $B$ bovis do occur, but are uncommon and evidently caused by a number of factors, including immune responsiveness of the vaccinated animals, and immunogenicity of the vaccine strain.

Immunity to Anaplasma marginale - The vaccine containing $A$ centrale provides partial, variable protection against $A$ marginale. Protection against challenge in Australia is adequate in most cases to prevent disease and use of the vaccine in this country appears to be justified. Protection against antigenically diverse, highly virulent stocks of $A$ marginale in other countries is, at times, clearly inadequate and better vaccines are required in situations where the challenge is severe.

Aust Vet J 2001;79:832-839

Key words Tick fever, Babesia bovis, Babesia bigemina, Anaplasma centrale, Anaplasma marginale, live cattle exports, vaccination.

\begin{tabular}{ll}
\hline PCR & Polymerase chain reaction \\
SE & South-east \\
NA & Not available \\
PCV & Packed cell volume \\
TFRC & Tick Fever Research Centre
\end{tabular}

$\mathrm{T}$ ick fever is an important livestock disease in Australia with more than 7 million cattle potentially exposed in endemic regions. ${ }^{1} \mathrm{M}$ cLeod and K ristjanson estimated that annual mortality associated with anaplasmosis and babesiosis in Australia in 1998 resulted in production losses of $\$ 22$ million despite spending on acaricides and vaccines of $\$ 8.5$ million. ${ }^{2}$ The disease caused by infections of Babesia bovis, $B$ bigemina and Anaplasma marginale is also a potential threat to the live cattle export trade to countries in SE Asia where these parasites are endemic. ${ }^{3}$

Although vaccines against tick fever have been used in Australia for up to a century, vaccines containing attenuated strains of $B$ bovis and B bigemina as well as A centrale grown in splenectomised calves have only been used in Australia since 1964 to immunise cattle against the disease. ${ }^{4}$ TFRC of the D epartment of Primary Industries, Q ueensland is the only provider of vaccine in Australia. Similar products are produced in South Africa, Argentina, Brazil, U ruguay and Israel. ${ }^{3}$ Both chilled and frozen vaccines are produced.

U se of tick fever vaccine in endemic parts of Australia is a risk management strategy based on the likelihood of exposure, the value and the susceptibility of the stock. In the live cattle export trade, pre-embarkation vaccination is, in some cases, a requirement for certain classes of cattle.

In total, about 800,000 doses of vaccine are supplied annually by TFRC and details of the production methods and standards have been published. ${ }^{5-7} T$ hese vaccines have shortcomings ${ }^{8}$ and questions are often asked about the degree of protection afforded, safety, viability and quality control.

The purpose of this paper is to provide some answers to questions relating to the protective immunity that follows use of Australian B bovis, B bigemina and A centrale vaccines. We have reviewed available literature and included unpublished field and experimental data. Where relevant, we have also used overseas experiences with similar products. We have focused more on clinical evidence of protection than on conventional immunological measures such as antibody and cellular responses. Finally, we make recommendations for practical prevention of tick fever in endemic parts of Australia and for the use of vaccines in cattle for export.

\section{Immunity to B bovis}

Immunity to heterologous challenge

Passively administered antibody provides protection against homologous but not heterologous strain challenge. ${ }^{9}$ Brown and Palmer ${ }^{10}$ concluded that inflammatory cell-mediated and cytophilic antibody responses were important in maintaining immunity during persistent infection or after exposure in immunologically primed animals particularly against heterologous challenge. Generally, a vaccine containing one attenuated strain of $B$ bovis provides protection against heterologous field challenge. Results of a field trial involving 179 vaccinated and 56 unvaccinated cattle on five Q ueensland properties showed that vaccinated heifers were 15 to 16 times less likely to contract babesiosis than unvaccinated ones. ${ }^{11} \mathrm{~T}$ his feature is worth noting because strains and isolates of $B$ bovis are known to be antigenically diverse, ${ }^{8,12,13}$ In fact, analysis of D N A extracts of 27 Australian isolates using two PCR assays showed that all differed from each other. ${ }^{14}$ Animals are also less susceptible to challenge with the same (homologous) strain than a different (heterologous) one..$^{15}$ In addition, animals exposed to more than one heterologous challenge are less susceptible to further challenges with other strains. D espite this, differences 
between isolates or strains do not appear to be important in field manifestations of disease and recovered animals usually withstand heterologous challenge. ${ }^{16}$ The $B$ bovis strain currently in use appears to provide excellent protection against a wide range of field isolates (Table 1 ) (Bock unpublished). There is also documented and circumstantial evidence that Australian B bovis vaccine protects against field isolates in Africa, ${ }^{17,18}$ South America ${ }^{19,20}$ and SE Asia. ${ }^{21}$

Since 1980, three strains of B bovis have been used at various times to produce vaccine (Figure 1 ), all attenuated by multiple syringe passage of a field isolate through splenectomised calves. ${ }^{22}$ It has been suggested that field isolates consist of subpopulations, each expressing virulence genes to varying degrees and that serial passage in splenectomised calves selects populations enriched with faster growing avirulent phenotypes. ${ }^{23}$

A change of strain used in the vaccine was generally made in response to field evidence of vaccine failures (Figure 1). Early immunisation strategies involved revaccination with at least one different vaccine strain. ${ }^{16} \mathrm{H}$ owever, it appears that the degree of protection resulting from the $B$ bovis vaccine is more closely related to the immunogenicity of the primary vaccine strain than the number of strains used. 24,25 As a result, advice to users is, in most cases, to vaccinate once only.

Some B bovis vaccine strains are less immunogenic than others. ${ }^{16,26}$ For example, there is evidence that multiple passaging of a strain may result in loss of immunogenicity (Table 2). ${ }^{25}$ Five clones of one vaccine strain (K) obtained by limiting dilution in vivo varied in their ability to protect against heterologous challenge, with a range of $50 \%$ to $90 \%$ as determined by PCV depression, compared to the parent strain. ${ }^{27}$ This suggests that a combination of different parasite populations is required for adequate protection. ${ }^{28}$ In a study of vaccine failures following the use of $T$ strain, 27 field isolates from 24 affected properties were analysed using PCR technology but no common parasite genotype was found that could be linked to the failures. ${ }^{14}$ D ixie strain was introduced in $M$ ay 1993 , first as a high passage (23 to 28 ) line containing one population characterised by PCR assay ${ }^{29}$ and, since January 1996, as a lower passaged (15 to 20) line with two populations. Both lines were protective against virulent challenge, although pen trials showed the protection provided by the low passaged line to be superior (Table 2). ${ }^{25} \mathrm{M}$ ore than 6 million doses of vaccine containing Dixie strain had been supplied by $D$ ecember 2000 and field evidence at that stage appeared to validate the protective effect seen in pen trials such as those listed in Table 1. The failure rate since its release fell within the acceptable range observed by Bock et al 24 for other protective strains (Figure 1).

Effect of virulence, dose rate and route of vaccination on immunity to challenge

$M$ ahoney et al 30 found no difference in the immunogenicity of virulent tick-transmitted B bovis parasites and an attenuated vaccine strain. Studies with clones of B bovis showed that protective immunity was unrelated to virulence with none of the clones giving as good protection as the parent strain. ${ }^{27}$ Using a limiting dilution method, Timms et al ${ }^{23}$ were able to infect splenectomised calves by the intravenous inoculation of one $B$ bovis parasite. H owever, De Vos ${ }^{26}$ showed that the infectivity of diluted vaccine parasites given subcutaneously became variable at $10^{5}$ with only three of five animals becoming infected, but when the dose was infective there was no

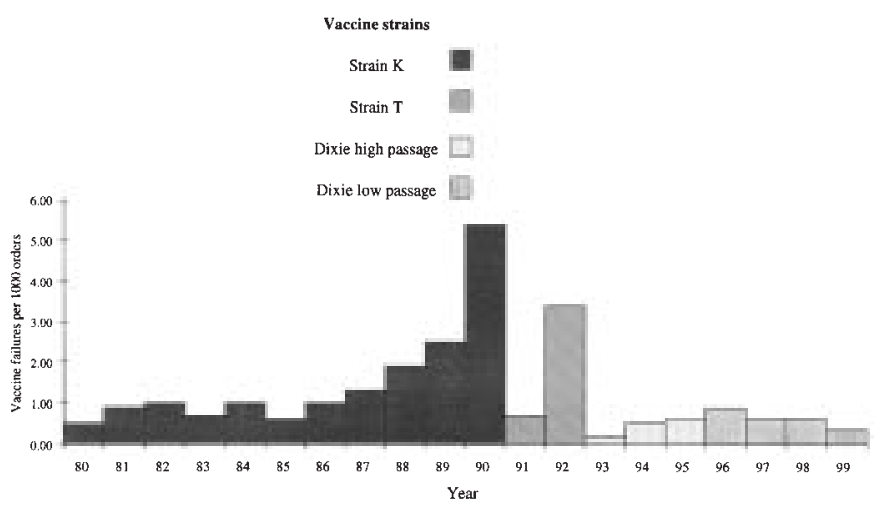

Figure 1. Herds with confirmed $B$ bovis vaccine failures related to vaccine supply and vaccine strain used from 1980 to 1999. A vaccine failure case was defined as: a confirmed diagnosis of infection at a QDPI laboratory; records showing vaccine was given to affected stock; evidence of seroconversion in vaccinated cohorts at the time of the outbreak. ${ }^{25}$ Failures per 1000 orders is used as an indication of the number of herds vaccinated as the case definition is based on herds not individual animals.

difference between the number of $B$ bovis organisms and the severity of the infections observed. ${ }^{4}$ At the $10^{7}$ dose of $B$ bovis used in the commercial vaccine it was fully infective when given intramuscularly or subcutaneously (Table 5). The effect of vaccine dose or route of inoculation on subsequent immunity has not been adequately addressed, but based on field evidence, the subsequent immunity appears to be independent of either provided the vaccine is infective.

\section{Duration of immunity}

$M$ ahoney and colleagues showed that the immunity conferred by a single tick-transmitted infection lasted for at least 4 years, the duration of their trial. ${ }^{30,31}$ Later, it was determined that the immunity after B bovis vaccination was comparable in strength and duration to that conferred by tick-transmitted infection. ${ }^{31}$ They found that cattle vaccinated as calves were still immune after 4 years in a tick-free area and showed no signs of clinical illness, fever or anaemia after heterologous challenge. In contrast, unvaccinated animals developed severe clinical signs and 4 of the 10 died. From these results, the authors concluded that a single vaccination of calves between 3 and 6 months of age would be sufficient to prevent clinical babesiosis even if tick populations were too low to maintain endemic stability. ${ }^{31}$

Immunity is known to persist after elimination of B bovis. Studies on cattle that are sterilised of the B bovis infection using drugs, suggested that the degree of acquired immunity was related more to the degree of antigenic stimulation experienced by the host than the presence of live parasites. ${ }^{12}$

Analysis of 32 confirmed reports of B bovis vaccine failures between 1968 and 1971 showed that the likelihood of failure did not increase with time after vaccination and 29 of 32 cases studied occurred within 6 months of vaccination. ${ }^{16} \mathrm{~A}$ subsequent study of failures between 1973 and 1990, a period when more than 20 million doses (300,000 orders) of vaccine were supplied, also showed no evidence of a time-dependant loss of immunity after vaccination. ${ }^{24}$ In one particular outbreak, affected cattle were vaccinated 10 months to 7 years before the 
outbreak with the same vaccine strain. More recently, Bock et al 25 studied 62 vaccine failures that occurred after use of $T$ strain between 1990 and 1993, and found the average time from vaccination to an outbreak to be 14 months (range 3 to 31 months).

In M alawi, an outbreak of B bovis in cattle vaccinated 2 years earlier with an Australian strain (K) was ascribed to a decline in immunity in the absence of tick challenge. ${ }^{32}$ H owever, the study design was such that other contributing factors, including vaccine infectivity and immunogenicity could not be excluded and we find it impossible to support the authors' conclusion of time-dependent immunity based on the evidence they provided.

$\mathrm{H}$ ost and related factors influencing the development of protective immunity

Age - The resistance of calves to primary B bovis infection is well known and is due to both passive and innate factors. ${ }^{8}$ Specific antibodies transferred via colostrum are important during the first 2 months and non-specific immunity provides some protection during the next 5 months at least. ${ }^{12}$ Advice to cattle owners in Australia is therefore to vaccinate their animals when 3 to 6 months of age when this non-specific immunity is greatest and the risk of reactions to the vaccine very small. We could find no experimental or other evidence to suggest that vaccination at this young age or even younger had any detrimental effect on subsequent immunity to challenge.

Stress - This can potentially have an effect on the immunity to tick fever. It is known that the immunity of cattle can be depressed experimentally in a number of ways, including surgical procedures (removal of the spleen), use of chemical immunosuppressants and concurrent infections.8,12 H owever, there is little field evidence that stress, including nutritional stress, has a significant effect on immunity following $B$ bovis vaccination. Losses to tick fever in almost 3500 naïve, mostly drought affected cattle were almost negligible (about 0.05\%) after vaccination and exposure to ticks for up to 11 months, despite the reported incidence of the disease in local cattle at the time being one of the highest on record. 33

Concurrent infections/vaccinations - A wide range of infective agents including viruses, parasites and bacterial diseases can suppress the immune responses of animals to other agents. ${ }^{34}$ $\mathrm{H}$ owever, little is known of the effect of other infections or concurrent use of other vaccines on the subsequent immunity of cattle following $B$ bovis vaccination.

H ost genotype - C ertain breeds of cattle, notably Bos taurus breeds, are known to be more susceptible to primary B bovis infection. ${ }^{35}$ Purebred $B$ indicus cattle are highly resistant to babesiosis, but crossbred cattle are sufficiently susceptible to warrant the use of preventive measures such as vaccination. ${ }^{36}$ Similarly, genotype also plays a role in the development of protective immunity with $B$ taurus breeds more likely to show a deficient immunity. An investigation of 62 reports of $T$ strain vaccine failures in $\mathrm{Q}$ ueensland showed that all were in cattle with no greater than $3 / 8 \mathrm{~B}$ indicus infusion and $85 \%$ were in pure $B$ taurus herds. ${ }^{25}$

\section{Investigation of vaccine failures}

Vaccine failures following the use of $B$ bovis vaccine undoubtedly occur in Australia (Figure 1). Bock et al ${ }^{25}$ defined a vaccine failure as: a confirmed diagnosis of B bovis infection at a QDPI laboratory; records showing vaccine was given to affected stock; evidence of seroconversion in vaccinated cohorts at the time of the outbreak. They considered eight possible factors, some of which were mentioned in the preceding discussion. While the situation is complex and no simple explanation is forthcoming, recent research emphasis has fallen on the immune responsiveness of the host.

\section{Immunity to Babesia bigemina}

Immunity to heterologous challenge

$C$ attle recovered from $B$ bigemina infections show some resistance to $B$ bovis challenge, ${ }^{37}$ but the reverse is not true and the degree of protection is seldom adequate. It is assumed that similar immune mechanisms to $B$ bovis exist in $B$ bigemina 
infections. ${ }^{10}$ Immunological differences between isolates and strains of $B$ bigemina are well known. Callow ${ }^{38}$ showed that self-cured, splenectomised calves resisted challenge with a homologous strain and, in some cases, even prevented detectable reestablishment of the infection, whereas the infection was almost invariably acute after heterologous challenge. A parallel study in intact steers confirmed the difference between the two strains with detectable parasitaemias developing only in some steers after homologous and in all steers after heterologous challenge.

$B$ bigemina is not an important cause of disease in Australia and accounted for only about $7 \%$ of all recorded outbreaks in Q ueensland between 1990 and $1996 .{ }^{39}$ It has also been estimated to cause $5 \%$ of the economic loss due to tick fever. ${ }^{5} \mathrm{~T}$ his is probably due to the greater prevalence of $\mathrm{B}$ bigemina infection (and therefore greater chance of endemic stability) and the relatively low pathogenicity of stocks present in this country. ${ }^{40}$ Despite the immunological differences amongst isolates, there is no evidence that variable antigenicity is an important factor in the natural disease. ${ }^{41}$ In general, cattle have one clinical infection and are then protected against subsequent challenge.

O nly one strain of B bigemina ( $G$ strain) has been used for production of vaccine in Australia since the 1980s. Its isolation and subsequent attenuation has been recorded. ${ }^{42}$ Laboratory evidence (Table $3 a, b, c)$ suggests that, while $B$ bigemina is generally of low pathogenicity, the strain affords sound protection, even when virulent laboratory isolates are used. 42,43 D emand for B bigemina vaccine was negligible before 1990 and field evaluation until that time was therefore impractical. Since then, demand has increased and more than 3.3 million doses were supplied between 1990 and 2000. The reasons for this appear to be more to do with availability, marketing and risk aversion because occurrence of the disease did not increase over this period.

Little documented evidence is available on the protection afforded by $G$ strain overseas. Pen trials in South Africa and Zimbabwe showed that it conferred adequate protection against local isolates (Table $3 d$, e). ${ }^{44,45,46}$ Results of a comparable trial in Paraguay were not clear-cut, mainly due to the low pathogenicity of the local strain used in challenge. ${ }^{19}$ Based on field use of this strain in South Africa, ${ }^{45}$ Zimbabwe ${ }^{47}$ and $\mathrm{M}$ alawi, 48 protection in these countries is evidently satisfactory. Three of 28 vaccinated cattle on smallholder properties in M alawi were clinically affected after challenge but the cause was not identified. ${ }^{48}$

Effect of virulence, dose rate and route of vaccination on immunity to challenge

The $\mathrm{G}$ strain of B bigemina has been the only commercial vaccine strain used in Australia since 1980 and we could find no published information on the role of virulence of the vaccine
Table 4. Responses of experimental cattle vaccinated with $\boldsymbol{A}$ centrale when challenged with different isolates of $\boldsymbol{A}$ marginale.

\begin{tabular}{lll}
\hline Group Parasitaemia (\%) & $\begin{array}{l}\text { Mean max PCV } \\
\text { depression (\%) }\end{array}$ & No. treated
\end{tabular}

a) Australia: Dayboro isolate -H59 (Bock unpublished 1997)

$\begin{array}{lrrr}\text { Vaccinated } & 4.1 & 24.9 & 0 / 5 \\ \text { Unvaccinated } & 12.7 & 49.7 & 4 / 5\end{array}$

b) Australia: Gypsy Plains -H67 (Bock unpublished 2000)

$\begin{array}{llll}\text { Vaccinated } & 0.7 & 14.5 & 0 / 6\end{array}$

$\begin{array}{llll}\text { Unvaccinated } & 7.0 & 37.8 & 4 / 7\end{array}$

c) Australia: Mt Walker -H83 (Bock unpublished 2000)

$\begin{array}{llll}\text { Vaccinated } & 2.1 & 21.1 & 2 / 7\end{array}$

$\begin{array}{lll}\text { Unvaccinated } & 16.4 & 35.4\end{array}$

d) Kenya: EAM isolate (adapted from Kuttler62)

$\begin{array}{lrrr}\text { Vaccinated } & 0.5 & 14.3 & \mathrm{NA} / 14\end{array}$

$\begin{array}{llll}\text { Unvaccinated } & 10.0 & 54.2 & \mathrm{NA} / 7\end{array}$

e) South Africa: BW isolate ${ }^{63}$

$\begin{array}{llll}\text { Vaccinated } & 16.7 & 51.0 & \mathrm{NA} / 13\end{array}$

$\begin{array}{llll}\text { Unvaccinated } & 32.0 & 60.8 & \mathrm{NA} / 5\end{array}$

f) Zimbabwe: $M$ isolate ${ }^{61}$

$\begin{array}{llll}\text { Vaccinated } & 21.4 & 61.4 & 6 / 9\end{array}$

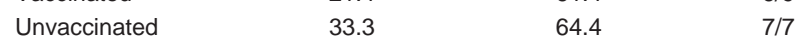

g) Argentina: Local isolate ${ }^{58}$

$\begin{array}{llll}\text { Vaccinated } & 2.3 & 32.3 & 0 / 12\end{array}$

$\begin{array}{lll}\text { Unvaccinated } & 7.5 & 50.0\end{array}$

h) Paraguay: Local isolate ${ }^{19}$

$\begin{array}{lccc}\text { Vaccinated } & 5.7 & 41.2 & 0 / 7 \\ \text { Unvaccinated } & 15.95 & 59.8 & 0 / 6\end{array}$

NA information was not available 
parasites and subsequent immunity. G strain has been shown to be infective subcutaneously at doses of $5 \times 10^{4}$ parasites with a decrease in prepatent period of approximately 1 day for each log increase in dose to $5 \times 10^{8}$ (Standfast N F, unpublished 1998). The dose currently used in the Australian commercial vaccine is $5 \times 10^{6}$ parasites and at this dose there was no apparent difference in infectivity between intramuscular and sub-cutaneous inoculations (Table 5). As for B bovis, the effect of vaccine dose or route of inoculation on subsequent immunity has not been adequately addressed, but provided the vaccine is infective the subsequent immunity also appears to be independent of either.

\section{D uration of immunity.}

Experimental animals naturally infected with $\mathrm{B}$ bigemina during calfhood and then kept tick-free for 4 years, all suppressed the parasitaemia upon reinfection with a heterologous strain in Q ueensland. ${ }^{31}$ The results of this trial suggest that a single infection during calfhood will provide immunity lasting at least 4 years. No detailed study of the duration of immunity following vaccination has been conducted in Australia mainly because of the lack of evidence of any deficiency in immunity following vaccination for whatever reason. It has been reported in South Africa that animals do become susceptible again in the absence of natural challenge and also that animals were susceptible 16 months after chemosterilisation of the infection. 45

$\mathrm{H}$ ost and related factors influencing the development of protective immunity

Age and breed effects ${ }^{35,49}$ influence the susceptibility of cattle to primary infections in much the same way they do with $B$ bovis. $\mathrm{H}$ owever, we could find no evidence in the literature to suggest that these or any other factors influenced the degree of immunity to subsequent challenge.

\section{Investigation of vaccine failures}

There is very little evidence that the $B$ bigemina vaccine does not protect in Australia. A review of routine diagnostic submissions to Q D PI laboratories between January 1993 to June 2000 identified only three cases of suspected vaccine failure that could be ascribed to immunogenicity of the vaccine (RE Bock unpublished). This low failure rate relative to $B$ bovis could, in part, be due to the low pathogenicity of most $B$ bigemina in this country.

\section{Immunity to Anaplasma marginale}

Immunity to heterologous challenge

The protective immune mechanism for anaplasma is not fully understood nor is the mechanism for establishment of persistent infection in the face of an immune response. Cattle recovered from acute infection or immunised with killed vaccines are solidly protected against challenge with the homologous A marginale strain, but are only partially protected against acute rickettsaemia after challenge with heterologous strains. ${ }^{50} \mathrm{Also}$, it has been concluded that although antibody alone is not sufficient for protection it is required to provide specificity for macrophage phagocytosis of infected erythrocytes. ${ }^{50}$ In
Australia, anaplasmosis accounted for slightly more than $10 \%$ of tick fever outbreaks between 1990 and 1996, and is rarely the cause of significant mortality. ${ }^{39}$

$O$ verseas, isolates of A marginale are known to exist which differ in antigenic composition, protection afforded against heterologous challenge, virulence and tick transmissibility. ${ }^{51-53}$ Although Australian isolates have not been studied closely, there is no clinical evidence to suggest that isolates in this country differ significantly in either antigenicity or virulence. C ross immunity testing of two A marginale isolates obtained $700 \mathrm{~km}$ apart in $\mathrm{N}$ orth Q ueensland were immunologically identical. ${ }^{54}$ The reason why the situation in Australia differs from that in other countries is unknown but may be related to a very limited number of variant stocks of $A$ marginale brought into this country when the vector Boophilus microplus was introduced during the 19th century. 55

Vaccine used in Australia contains A centrale, an organism imported from South Africa in 1934.56 This organism was originally isolated in 191157 and is used in vaccine produced in a number of countries, including South Africa, ${ }^{57}$ Argentina, ${ }^{58}$ U ruguay, ${ }^{59}$ Israel, 60 and, more, recently also Zimbabwe ${ }^{61}$ and M alawi. ${ }^{48}$

Immunity imparted by A centrale against challenge with Australian isolates of A marginale is partial and variable (Table $4 a, b, c)$. D espite the incomplete protection, field evidence collected from diagnostic laboratory statistics suggests that it is adequate to control the effects of $A$ marginale infections in this country.

Evidence of the protective potential of $A$ centrale in other countries is very variable. In Kenya, vaccination resulted in a significant reduction in the severity of experimental $A$ marginale challenge (Table 4d). ${ }^{62}$ In South Africa and in Zimbabwe, feedback from vaccine users has generally been positive but the results of some pen trials involving challenge with virulent isolates have been discouraging. Potgieter and van Rensburg 63 in South Africa found the protection in some animals to be poor (Table 4e), even after a second vaccination. They believed this could be due to high challenge doses used in their trials and the virulence of the isolate. $\mathrm{H}$ owever, in an A marginale challenge of naïve $\mathrm{H}$ ereford steers in Australia, it was found that, whereas the mean time taken to reach a $1 \%$ parasitaemia was $7.3,13.9$ and 19.9 days in the $10^{10}, 10^{8}$ and $10^{6}$ infection dose groups, respectively, the results showed that the pathological outcomes (anaemia) of anaplasmosis were similar over the 10,000-fold infective dose range tested. ${ }^{64}$ In Zimbabwe, no significant differences were found when vaccinated and unvaccinated groups were challenged with a high dose of a virulent field 
isolate (Table 4f). ${ }^{61}$ Even use of different stocks of A centrale originating from South Africa and Australia did not influence the results.

Similar variable results have been described in South America. In Argentina, the degree of protection afforded by A centrale was considered to be reasonable (Table $4 \mathrm{~g})^{58}$ whereas, in pen (Table 4h) and field trials in Paraguay, it was insufficient. ${ }^{19,} 65$

We could find no reports on the protection afforded by $A$ centrale in Asia even though vaccine has been used to protect cattle imported into a number of countries, especially in SE Asia.

The mechanism of protective immunity provided by $A$ centrale is unclear but may be induced by cross-reactive epitopes on immunodominant antigens shared by $A$ centrale and $A$ marginale. 66, 67 The cross-protection appears to be unidirectional with $A$ marginale not providing any protection against $A$ centrale ${ }^{62}$

Effect of virulence, dose rate and route of vaccination on immunity to challenge

O nly one strain of A centrale is used in the vaccine and no observations can therefore be made on the effect of virulence on immunogenicity. In A marginale, however, strains differ in virulence and ability to afford protection against heterologous challenge. A buffalo-derived A marginale isolate in South Africa proved to be less virulent than $A$ centrale, but afforded even less protection against a virulent $A$ marginale challenge. ${ }^{63} T$ he route of inoculation can have a marked effect on the infectivity of the vaccine, but at the doses used in the commercial chilled vaccine there does not appear to be any difference between intramuscular and subcutaneous routes (Table 5).

\section{Duration of immunity}

Animals inoculated with A centrale remain infected for a long time, probably for life, ${ }^{68}$ and there is no evidence that immunity wanes with time. An A centrale specific ELISA developed by M olloy et al ${ }^{69}$ detected antibodies to A centrale in cattle up to 9 years after vaccination with no apparent decrease in test sensitivity. Field evidence from some properties where the vaccine has been used for many years, suggested that natural transmission of A marginale has ceased (Bock unpublished observations).

Little information is available on the persistence of immunity following drug elimination of $A$ centrale but it is likely to parallel that of A marginale infection where immunity persisted for a year (the duration of the trial) after elimination of the infection with imidocarb. ${ }^{71}$

$\mathrm{H}$ ost and related factors influencing the development of protective immunity

Age - N on-specific resistance of calves to anaplasmosis is well known ${ }^{72}$ and resembles that of $B$ bovis infection. It has been suggested that cattle vaccinated at a young age may experience such mild reactions that the immune response is insufficient. ${ }^{5}$ $\mathrm{H}$ owever, there is no field evidence to suggest that vaccination of young animals is not effective in Australia. ${ }^{54}$

Stress- Immune-compromised animals, including removal of the spleen and use of immunosuppressants such as cyclophosphamide and corticosteroids, have been shown experimentally to be susceptible to heterologous challenge. ${ }^{73}$ It has also been suggested that the immunity of cattle could be compromised under conditions of environmental stress or other stressors ${ }^{73}$ but we could find no published evidence of this being a significant factor under field conditions. In fact, W ilson 72 showed that cattle on a good plane of nutrition developed more severe primary Anaplasma reactions than those on a starving ration, but he did not observe the effect on subsequent immunity.

Concurrent infections or vaccinations and genotype - We could find no evidence that concurrent infections or vaccinations and breed have an effect on the development or level of immunity to A centrale. Bos indicus cattle do not seem to show the same degree of natural resistance to primary Anaplasma infections as is seen with, for instance, B bovis. ${ }^{35,} 72,74$ Reports of variable protection conferred by A centrale (Table 4), involved Bos taurus, Bos indicus and cross-bred cattle.

Investigation of vaccine failures

The occurrence of vaccine failures in cattle vaccinated with $A$ centrale in Australia is insignificant. ${ }^{16}$ We have investigated some reports of failures and found the main cause to be poor viability rather than a lack of immunogenicity of the vaccine (Bock et al unpublished observations). The problem was corrected by reducing the use-by date of the vaccine and promoting its proper handling, but infectivity remains lower for $A$ centrale than the Babesia parasites (Table 5).

In 1997, we investigated a suspected breakthrough isolate (D ayboro) obtained from a property where clinical cases were seen in vaccinated cattle (Bock unpublished observations). Experimental challenge of vaccinated cattle with this isolate (Table 4a) showed that three of the five cattle had adequate immunity while one had a reaction almost comparable with those of the susceptible controls. This variability in individual responses has been seen before: Wilson et al ${ }^{54}$ found that 10 of 18 cattle challenged with another Australian isolate had inadequate immunity whereas the remainder were solidly protected.

Field observations of apparent vaccine failure on another property ( $\mathrm{M}$ t Walker, SE Q ueensland) are being investigated, and while the strain is very virulent (Table 4c) there appear al so to be herd or animal problems involving the ability of cattle to mount a protective response.

0 verseas, overt disease was reported in vaccinated cattle on a property in Zimbabwe during evaluation of $A$ centrale vaccine. ${ }^{61}$ As discussed in a previous section, pen trials showed that the vaccine afforded very little protection against an isolate obtained from this property. Interestingly, commercial non-living vaccine (Plasvax ${ }^{\circledR}$ ) also failed to protect cattle against this isolate in a separate pen trial (M atingo unpublished observations 1997).

\section{Recommendations for use of B bovis, B bigemina and $A$ centrale vaccines in Australia}

In endemic areas

A single vaccination at 6 to 9 months of age should provide strong, lasting protection. At that age, the risk of vaccine reactions is minimal. There is no evidence that immunity wanes with time and revaccination is therefore unnecessary as a routine procedure. Revaccination is advisable when there is uncertainty over the accuracy of previous procedures, to ensure all animals seroconvert (Table 5) or when there has been a change in the strains used in the vaccine. ${ }^{24,25}$

Australian cattle owners have a choice of two vaccines: They can either vaccinate their cattle with a trivalent vaccine containing all three organisms, or a bivalent one containing $B$ bovis and $A$ centrale. Trivalent vaccine is most often 
recommended for B os taurus breeds, valuable animals such as stud cattle, and cattle originating from tick-free properties. Bivalent vaccine is most often used in Bosindicus and cross-bred cattle born in tick-infested areas. $35,36,49,74$

\section{In live cattle export trade}

Current recommendations vary depending on the requirements of the importing country, the origin of the cattle (endemic or non-endemic regions) and the type of cattle (breeders or cattle destined for feedlots). Where import requirements stipulate vaccination, it is usually to be done very shortly before export.

Based on the evidence provided in this review, vaccination at weaning age ( 6 to 9 months) will provide adequate protection after export. This will negate the need to muster and hold cattle some time before export and also reduce the risk of vaccine reactions en route. The savings to exporters are therefore likely to be significant if animals are vaccinated at an early age. The main proviso will be an on-farm quality assurance system that ensures proper use of the vaccine and identification of immunised animals and which is accepted by importing countries. Vaccination against B bovis, B bigemina and A centrale will ensure broad spectrum protection in most situations, but caution is required with regard to the immunity produced by $A$ centrale.

\section{The future}

The current live tick fever vaccine has a number of shortcomings including a short shelf-life, potential for cotransmission of infectious agents, animal welfare considerations, high cost of production and increasingly stringent national registration requirements. These limitations have led to continuing efforts to develop vaccines based on recombinant DN A technology. 10,50 In Australia this work has all but been discontinued. The major limitations such as research funding, variations in immune responsiveness ${ }^{75}$ and the need for repeat vaccinations mean that recombinant products cannot presently compete with the available live vaccine.

M uch of the evidence for the efficacy of the Australian tick fever vaccine is based on feedback from field use of over 30 million doses since 1966 because many of the 'pen trials' include only small numbers of animals. The shortcomings of the live vaccine can be minimised by good operating standards, efficient transportation and continuing research. Improvements in freezing and post-thawing viability of the frozen tick fever vaccine $^{76}$ as well as a better understanding of host-parasite immune interactions could overcome the problems associated with the live vaccine. For the next 5 to 10 years at least the live vaccine will be the basis for protection against these parasites in Australia.

\section{References}

1. Chudleigh P. Tick-borne disease control in cattle. In: Economic Assessment Series. Australian Centre for International Agricultural Research, Canberra, 1991;5:1-24.

2. McLeod R, Kristjanson P. Economic impact of ticks and tick-borne diseases to livestock in Africa, Asia and Australia. International Livestock Research Institute, Nairobi, 1999

3. de Castro JJ. Sustainable tick and tickborne disease control in livestock improvement in developing countries. Vet Parasitol 1997;71:77-97.

4. Callow LL, Dalgliesh RJ, de Vos AJ. Development of effective living vaccines against bovine babesiosis - the longest field trial? Int J Parasitol 1997;27:747-67.

5. Anonymous. Ticks and Tick-borne Disease Control: A Practical Field Manual. Vol II Tick-borne Disease Control. FAO, Rome, 1984.

6. de Vos AJ, Pipano E, Musisi F, Jorgensen WK. Protozoal and Rickettsial
Vaccines. In: Mowat G, Rweyemamu M, editors. Vaccine Manual - The production and quality control of veterinary vaccines for use in developing countries. FAO, Rome, 1997:75-89.

7. de Vos AJ, Jorgensen WK. Bovine babesiosis. In: Manual of Standards for Diagnostic Tests and Vaccines. Office International des Epizootic, Paris, 1996:333-338

8. de Vos AJ, Dalgliesh RJ, Callow LL. Babesia. In: Soulsby EJL, editor. Immune responses in parasitic infections: immunology, immunopathology and immunoprophylaxis. CRC Press, Boca Raton, 1987; III.

9. Mahoney DF. Immune responses to hemoprotozoa II. Babesia spp. In: Soulsby EJL, editor. Immunity to animal parasites. Academic Press, New York, 1972:301-341

10. Brown WC, Palmer GH. Designing blood-stage vaccines against Babesia bovis and B. bigemina. Parasitol Today 1999;15:275-281.

11. Emmerson FR, Knott SG, Callow LL. Vaccination with Babesia argentina in 5 beef herds in south-eastern Queensland. Aust Vet J 1976;52:451-454.

2. Dalgliesh RJ. Babesiosis. In: Warren SK, editor. Immunology and molecular biology of parasite infections. Blackwell, Oxford, 1993:352-383

13. Dalrymple B. Diversity and selection in Babesia bovis and their impact on vaccine use. Parasitol Today 1992;8:21-23.

14. Lew AE, Bock RE, Croft JM et al. Genotypic diversity in field isolates of Babesia bovis from cattle with babesiosis after vaccination. Aust Vet $J$ 1997;75:575-578.

15. Callow LL. A note on homologous strain immunity in Babesia argentina infections. Aust Vet J 1968;44:268-269.

16. Callow LL, Dalgliesh RJ. The development of effective, safe vaccination against babesiosis and anaplasmosis in Australia. In: Johnston LAY, Cooper MG, editors. Ticks and Tick borne Diseases. Proceedings of a Symposium held at the 56th Annual Conference of the Australian Veterinary Association, Townsville, 1980:4-8

17. de Vos AJ, Bessenger R, Fourie CG. Virulence and heterologous strain immunity of South African and Australian Babesia bovis strains with reduced pathogenicity. Onderstepoort J Vet Res 1982;49:133-136.

18. Lawrence JA, Malika J, Whiteland AP, Kafuwa P. Efficacy of an Australian Babesia bovis vaccine strain in Malawi. Vet Rec 1993:132:295-296.

19. Brizuela CM, Ortellado CA, Sanabria E, Torres O, Ortigosa D. The safety and efficacy of Australian tick-borne disease vaccine strains in cattle in Paraguay. Vet Parasitol 1998;76:27-41.

20. Callow LL, Quiroga QC, McCosker PJ. Serological comparison of Australian and South American strains of Babesia argentina and Anaplasma marginale. Int Parasitol 1976:6:307-310.

21. Callow LL. Tick-borne livestock diseases and their vectors. 3. Australian methods of vaccination against anaplasmosis and babesiosis. World Anim Rev 1976:18:9-15

22. Callow LL, Mellors LT, McGregor W. Reduction in virulence of Babesia bovis due to rapid passage in splenectomized cattle. Int J Parasitol 1979:9:333-338.

23. Timms P, Stewart NP, De Vos AJ. Study of virulence and vector transmission of Babesia bovis by use of cloned parasite lines. Infect Immun 1990:58:2171-2176.

24. Bock RE, de Vos AJ, Kingston TG, Shiels IA, Dalgliesh RJ. Investigations of breakdowns in protection provided by living Babesia bovis vaccine. Vet Parasitol 1992;43:45-56.

25. Bock RE, de Vos AJ, Lew A, Kingston TG, Fraser IR. Studies on failure of $T$ strain live Babesia bovis vaccine. Aust Vet J 1995;72:296-300.

26. de Vos AJ. Immunogenicity and pathogenicity of three South African strains of Babesia bovis in Bos indicus cattle. Onderstepoort J Vet Res 1978:45:119-124. 27. Timms P. Development of babesial vaccines. Trans $R$ Soc Trop Med Hyg 1989;83:73-79.

28. Gill AC, Cowman AF, Stewart NP, Kemp DJ, Timms P. Babesia bovis: molecular and biological characteristics of cloned parasite lines. Exp Parasitol 1987;63:180-188.

9. Lew AE, Dalrymple BP, Jeston PJ, Bock RE. PCR methods for the discrimination of Babesia bovis isolates. Vet Parasitol 1997;71:223-237.

30. Mahoney DF, Wright IG, Goodger BV. Immunity in cattle to Babesia bovis after single infections with parasites of various origin. Aust Vet J 1979:55:10-12. 31. Mahoney DF, Wright IG, Mirre GB. Bovine babesiasis: the persistence of immunity to Babesia argentina and B. bigemina in calves (Bos taurus) after naturally acquired infection. Ann Trop Med Parasitol 1973;67:197-203. 32. Tjornehoj KT, Lawrence JA, Whiteland AP, Kafuwa PT. Field observations on the duration of immunity in cattle after vaccination against Anaplasma and Babesia species. Onderstepoort J Vet Res 1996;63:1-5.

33. Meehan JF. Field proof for the tick fever vaccine. Qld Agric J 1969;95:768-771. 34. Tizzard IR. Veterinary Immunology - An introduction. 5th edn. Saunders, Philadelphia, 1996.

35. Bock RE, de Vos AJ, Kingston TG, McLellan DJ. Effect of breed of cattle on innate resistance to infection with Babesia bovis, $B$ bigemina and Anaplasma marginale [Published erratum appears in Aust Vet $J$ 1997;75:449]. Aust Vet $J$ 1997;75:337-340.

36. Bock RE, Kingston TG, de Vos AJ. Effect of breed of cattle on transmission 
rate and innate resistance to infection with Babesia bovis and $B$ bigemina transmitted by Boophilus microplus. Aust Vet $J$ 1999;77:461-464.

37. Wright IG, Goodger BV, Leatch G et al. Protection of Babesia bigeminaimmune animals against subsequent challenge with virulent Babesia bovis. Infect Immun 1987;55:364-368.

38. Callow LL. Sterile immunity, coinfectious immunity and strain differences in Babesia bigemina infections. Parasitology 1967:57:455-465.

39. Bock R, de Vos A, Rayner A et al. Assessment of the risk of tick fever mortalities in north-western Queensland beef industry. In: Challenging the boundaries, Proceedings of section of Annual Conference, Australian Association of Cattle Veterinarians. Australian Veterinary Association, Brisbane, 1997:175-182.

40. Callow LL. Some aspects of the epidemiology and control of bovine babesiosis in Australia. J S Afr Vet Assoc 1979;50:353-356.

41. Callow LL. Piroplasms. In: Animal Health in Australia, Protozoal and rickettsial diseases. Australian Bureau of Animal Health, AGPS, Canberra, 1984:5:121-160

42. Dalgliesh RJ, Callow LL, Mellors LT, McGregor W. Development of a highly infective Babesia bigemina vaccine of reduced virulence. Aust Vet J 1981;57:8-11.

43. Jorgensen WK, De Vos AJ, Dalgliesh RJ. Comparison of immunogenicity and virulence between Babesia bigemina parasites from continuous culture and from a splenectomised calf. Aust Vet $J$ 1989;66:371-372.

44. de Vos AJ, Combrink MP, Bessenger R. Babesia bigemina vaccine: comparison of the efficacy and safety of Australian and South African strains under experimental conditions in South Africa. Onderstepoort $J$ Vet Res 1982;49:155-158

45. de Waal D. Vaccination against babesiosis. Acta Parasit Turcica 1996:20:487-499.

46. Turton J, Katsande T. Bovine babesiosis and anaplasmosis in Zimbabwe. In: Proceedings of the 2nd Tick-Host-Pathogen Interface Conference, Berg-enDal, 1995:46.

47. Katsande TC, Turton JA. Progress on the production of frozen Anaplasma and Babesia whole blood vaccines at the Central Veterinary Laboratory in Harare. In: Proceedings of the Zimbabwe Veterinary Congress, Harare, Zimbabwe, 1995.

48. Tjornehoj K, Lawrence JA, Kafuwa PT, Whiteland AP, Chimera BA. Immunisation of smallholder dairy cattle against anaplasmosis and babesiosis in Malawi. Trop Anim Health Prod 1997:29:77-82.

49. Bock RE, Kingston TG, Standfast NF, de Vos AJ. Effect of cattle breed on innate resistance to inoculations of Babesia bigemina. Aust Vet J 1999;77:465-466. 50. Palmer GH, Rurangirwa FR, Kocan KM, Brown WC. Molecular basis for vaccine development against the ehrlichial pathogen Anaplasma marginale. Parasitol Today 1999:15:281-286.

51. Palmer G. Anaplasma vaccines, In: Wright I, editor. Veterinary Protozoan and Hemoparasite Vaccines. CRC Press, Boca Raton, 1989:1-29.

52. Eriks IS, Stiller D, Goff WL et al. Molecular and biological characterization of a newly isolated Anaplasma marginale strain. J Vet Diagn Invest 1994;6:435-441. 53. Tebele N, Palmer GH. Crossprotective immunity between the Florida and a Zimbabwe stock of Anaplasma marginale. Trop Anim Health Prod 1991;23:197-202.

54. Wilson AJ, Parker R, Trueman KF. Experimental immunization of calves against Anaplasma marginale infection: observations on the use of living $A$ centrale and A marginale. Vet Parasitol 1980;7:305-311.

55. Angus B. The history of the cattle tick Boophilus microplus in Australia and achievements in its control. International Journal of Parasitology 1996;26:1341-1355. 56. Rogers RJ, Shiels IA. Epidemiology and control of anaplasmosis in Australia. J S Afr Vet Assoc 1979;50:363-366.

57. Potgieter FT. Epizootiology and control of anaplasmosis in South Africa. JS Afr Vet Assoc 1979;50:367-72.
58. Anziani OS, Tarabla HD, Ford CA, Galleto C. Vaccination with Anaplasma centrale: response after an experimental challenge with Anaplasma marginale. Trop Anim Health Prod 1987;19:83-87.

59. Nari A, Solari M, Cardozo H. Hemovacuna para el control de Babesia spp. y Anaplasma marginale en el Uruguay. Veterinaria (Montevideo, Uruguay) 1979;15:137-148.

60. Pipano E, Krigel Y, Frank M, Markovics A, Mayer E. Frozen Anaplasma centrale vaccine against anaplasmosis in cattle. Br Vet $J$ 1986;142:553-556. 61. Turton JA, Katsande TC, Matingo MB et al. Observations on the use of Anaplasma centrale for immunization of cattle against anaplasmosis in Zimbabwe. Onderstepoort J Vet Res 1998;65:81-86.

62. Kuttler KL. A study of the immunological relationship of Anaplasma marginale and Anaplasma centrale. Res Vet Sci 1967;8:467-471.

63. Potgieter FT, Van Rensburg L. Infectivity virulence and immunogenicity of Anaplasma centrale live blood vaccine. Onderstepoort J Vet Res 1983;50:29-31. 64. Gale KR, Leatch G, DeVos AJ, Jorgensen WK. Anaplasma marginale: effect of challenge of cattle with varying doses of infected erythrocytes. Int $J$ Parasitol 1996;26:1417-1420

65. Payne RC, Osorio O, Ybanez A. Tick-borne diseases of cattle in Paraguay. II. Immunisation against anaplasmosis and babesiosis. Trop Anim Health Prod 1990;22:101-108.

66. Adams JH, Shiels IA, de Vos AJ. Heterologous antibody responses of calves to Anaplasma centrale and A. marginale. Vet Parasitol 1989;31:7-12. 67. Shkap V, Pipano E, McGuire TC, Palmer GH. Identification of immunodominant polypeptides common between Anaplasma centrale and Anaplasma marginale. Vet Immunol Immunopathol 1991;29:31-40.

68. Krigel Y, Pipano E, Shkap V. Duration of carrier state following vaccination with live Anaplasma centrale. Trop Anim Health Prod 1992;24:209-210.

69. Molloy JB, Bock RE, Templeton JM et al. Monoclonal antibodies differentiate between the Australian vaccine strain of $A$. centrale and $A$. marginale: Development of a fluorescent antibody test specific for $A$. centrale and an ELISA for the serological identification of vaccinated cattle. Int $J$ Parasitol 2001;31:179-186.

70. Wright IG, Leatch G. Bovine anaplasmosis. In: Manual of standards for diagnostic tests and vaccines. Office International des Épizooties, Paris, 1996:295-304.

71. Roby TO, Amerault TE, Mazzola V, Rose JE, llemobade A. Immunity in bovine anaplasmosis after elimination of Anaplasma marginale infections with imidocarb. Am J Vet Res 1974;35:993-995.

72. Wilson AJ. Observations on the pathogenesis of anaplasmosis in cattle with particular reference to nutrition, breed and age. J S Afr Vet Assoc 1979;50:293-295. 73. Kuttler KL, Zaugg JL, Johnson LW. Serologic and clinical responses of premunized, vaccinated, and previously infected cattle to challenge exposure by two different Anaplasma marginale isolates. Am J Vet Res 1984;45:22232226.

74. Bock RE, Kingston TG, de Vos AJ. Effect of breed of cattle on innate resistance to infection with Anaplasma marginale transmitted by Boophilus microplus. Aust Vet J 1999;77:748-751.

75. Court RA, Sitte K, Opdebeeck JP, East IJ. Mapping the T cell epitopes of the Babesia bovis antigen 12D3: implications for vaccine design. Parasite Immunol 1998;20:1-8.

76. Dalgliesh RJ, Jorgensen WK, de Vos AJ. Australian frozen vaccines for the control of babesiosis and anaplasmosis in cattle-a review. Trop Anim Health Prod 1990;22:44-52.

77. Callow LL, Pepper PM. Measurement of and correlations between fever, changes in the packed cell volume and parasitaemia in the evaluation of the susceptibility of cattle to infection with Babesia argentina. Aust Vet $J$ 1974;50:1-5.

(Accepted for publication $21 \mathrm{M}$ ay 2001) 